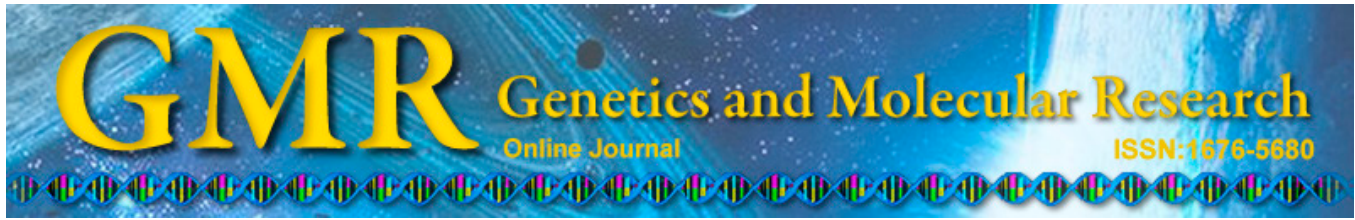

\title{
Association of the p53 Arg72Pro polymorphism with esophageal cancer in Chinese populations: a meta-analysis
}

\author{
J.Z. Peng ${ }^{1}$, L. Xue ${ }^{2}$, D.G. Liu' ${ }^{1}$ and Y.H. Lin ${ }^{3}$ \\ ${ }^{1}$ Department of Cardiothoracic Surgery, \\ The Third Affiliated Hospital of Southern Medical University, Guangzhou, \\ China \\ ${ }^{2}$ Department of Cardiothoracic Surgery, Guang Zhou Red Cross Hospital, \\ Guangzhou, China \\ ${ }^{3}$ Department of Neurology, \\ The Third Affiliated Hospital of Southern Medical University, Guangzhou, \\ China
}

Corresponding author: Y.H. Lin

E-mail: Linyihui68@126.com

Genet. Mol. Res. 14 (3): 9024-9033 (2015)

Received December 29, 2014

Accepted April 23, 2015

Published August 7, 2015

DOI http://dx.doi.org/10.4238/2015.August.7.11

\begin{abstract}
Although many epidemiologic studies have investigated the p53 codon 72 polymorphism and its association with esophageal cancer (EC) in China, definite conclusions could not be drawn. To clarify the effects of p53 codon 72 polymorphism on the risk of EC, we performed a meta-analysis on the Chinese population. A total of 13 studies including 3308 patients and 5115 controls were involved in this meta-analysis. Our results suggested that the Pro/Pro genotype was associated with an increased risk of EC in the Chinese population (Pro vs Arg: odds ratio $(\mathrm{OR})=1.17,95 \%$ confidence interval $(\mathrm{CI})=1.01$ 1.34; Pro/Pro vs Arg/Arg+Arg/Pro: OR = 1.32, 95\%CI = 1.06-1.66; Pro/ Pro vs Arg/Arg: $\mathrm{OR}=1.35,95 \% \mathrm{CI}=1.03-1.78$; Pro/Pro vs Arg/Pro:
\end{abstract}


$\mathrm{OR}=1.32,95 \% \mathrm{CI}=1.06-1.65)$. In subgroup analyses by geographical location and ethnicity, significant association was also found in south China in both the Han Chinese population and in the population group with no stated ethnicity. Sensitivity analysis confirmed the reliability and stability of the meta-analysis. No publication bias was found among studies using the Egger test. In conclusion, this meta-analysis provides supporting evidence that the Pro/Pro genotype might contribute to the development of EC in the Chinese population, especially in Southern China.

Key words: Meta-analysis; P53 codon 72; Polymorphism; Esophageal cancer

\section{INTRODUCTION}

Esophageal cancer (EC) is one of the most prevalent cancers in the world, with an estimated 482,300 new cases and 406,800 deaths in 2008 alone (Jemal et al., 2011). Its incidence rates vary internationally, and the highest rates found in Southern and Eastern Africa and Eastern Asia were nearly 16-fold higher than the lowest rates observed in Western and Middle Africa and Central America in both men and women (Jemal et al., 2011). In China, EC is the fourth most frequently diagnosed cancer and is the fourth leading cause of cancer deaths, with an estimated 259,235 new cases and 211,084 deaths in 2008 (Wiseman, 2008; Lin et al., 2013). It has been reported that EC is a multi-stage process, identified in most patients in the later period, and with a 5-year survival rate less than 10\% (Huang et al., 2013). Early detection and treatment is critical for the prevention of EC. Toward this goal, epidemiological studies have suggested that genetic factors such as TP53 (p53) might contribute to the development of EC.

p53 is one of the most extensively studied genes with respect to its role as a tumor suppressor. It has been considered to play a pivotal role in modulating cell growth, division, and apoptosis. Mutant p53 might contribute to increased cell proliferation, loss of ability to undergo apoptosis, and increasing genetic instability (Levine, 1997). An important $p 53$ polymorphism is the restriction fragment length polymorphism in codon 72 of exon 4, encoding proline (72Pro: CCC) or arginine (72Arg: CGC) (Dumont et al., 2003). The two structural forms have been shown to have differing biochemical and biological properties (Thomas et al., 1999), such as binding to components of the transcriptional machinery and activation of transcription (Langerod et al., 2002).

A number of studies have been conducted in China to explore whether the p53 codon 72 polymorphism is associated with EC susceptibility; however, these studies have provided controversial or inconclusive results. Therefore, we conducted a meta-analysis to define more precisely the effect of p53 codon 72 polymorphism on the risk of EC in Chinese populations.

\section{MATERIAL AND METHODS}

\section{Materials}

We searched PubMed, the Chinese Biomedical Database (CBM), and Chinese National Knowledge Infrastructure (CNKI) to collect related articles on the association between 
p53 codon 72 polymorphism and the susceptibility to EC published prior to April 4, 2014. References of the retrieved articles were also screened for additional original studies. The following search terms were used: "TP53 or p53" and "esophagus or esophageal" and "cancer or carcinoma" and polymorphism.

\section{Eligibility criteria}

Studies included in our meta-analysis must have met the following criteria: 1) used case-control or cohort study design; 2) provided the distribution of $\mathrm{p} 53$ codon 72 polymorphic alleles; 3) the gene distributions of the control groups were in agreement with Hardy-Weinberg equilibrium (HWE); 4) in Chinese; and 5) meta-analyses, letters, case reports, reviews, or editorial articles were excluded; and 6) if there were duplicate reports, the study with the largest number of patients was included.

\section{Data extraction}

The following information was extracted from each study: authors, year of publication, geographical location, ethnicity of participants, source of controls, number of patients and controls, genotype frequencies, and HWE test. The categorization of ethnicity comprised Han and other ethnic Chinese. For articles including different ethnicities, data were extracted separately for each ethnicity (Table 1).

\section{Statistical analysis}

Pooled odds ratios (ORs) and 95\% confidence intervals (CIs) were used to assess the strength of the associations. We also calculated pooled ORs and $95 \%$ CIs for all studies combined. Furthermore, subgroup analyses were performed by ethnic group and by geographical location. HWE in the control group for all studies was evaluated using the chi-square test. Statistical heterogeneity among studies was estimated by use of the $\mathrm{Q}$ and $\mathrm{I}^{2}$ statistic (Higgins and Thompson, 2002). A P value greater than 0.10 for the Q-test indicated a lack of heterogeneity among studies. Depending on the results of the heterogeneity test among individual studies, the fixed-effect (Mantel-Haenszel) or random-effect (DerSimonian and Laird) model was selected to summarize the pooled OR. A sensitivity analysis was performed to illustrate the accuracy and stability of the analytic results. Publication bias was investigated with a funnel plot, in which the standard error of the logOR of each study was plotted against its OR. Funnel-plot asymmetry was further assessed using the Egger linear regression test. All P values were two sided. A P value less than 0.05 was considered to be statistically significant. All statistical analysis was conducted by using Stata version 10.0 (StataCorp, College Station, TX, USA).

\section{RESULTS}

\section{Eligible studies}

To include all of the published articles, we used MeSH terms and Entry terms as the retrieval strategy. Eventually, 13 case-control studies (12 articles) (Lee et al., 2000; Peixoto Guimaraes et al., 2001; Li et al., 2002; Zhang et al., 2002, 2003; Hu et al., 2003; Hong et al., 
2005; Cai et al., 2006; Cao et al., 2007; Shao et al., 2008; Zhang, 2008; Li, 2012) were included and 176 articles were excluded. The publication years of the included studies ranged from 2000 to 2012. A flow chart of the study selection is shown in Figure 1. In total, 3308 patients with EC and 5115 controls were involved in this meta-analysis, which evaluated the relationship between p53 codon 72 polymorphism and EC risk. All studies were population based; five reported the same race (Han Chinese) and eight did not mention ethnicity. The characteristics of the included studies are summarized in Table 1.

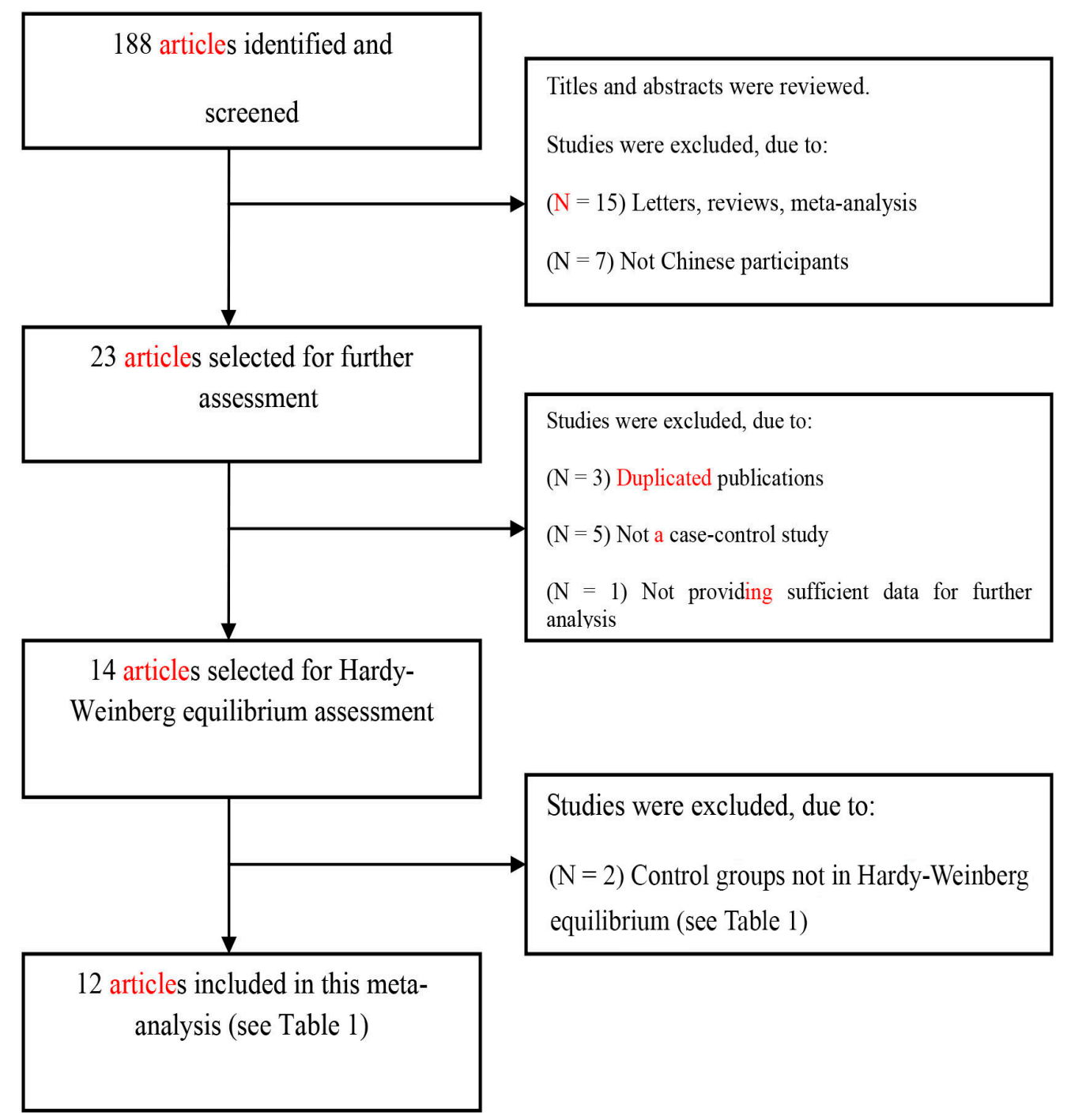

Figure 1. Article identification and exclusion. 


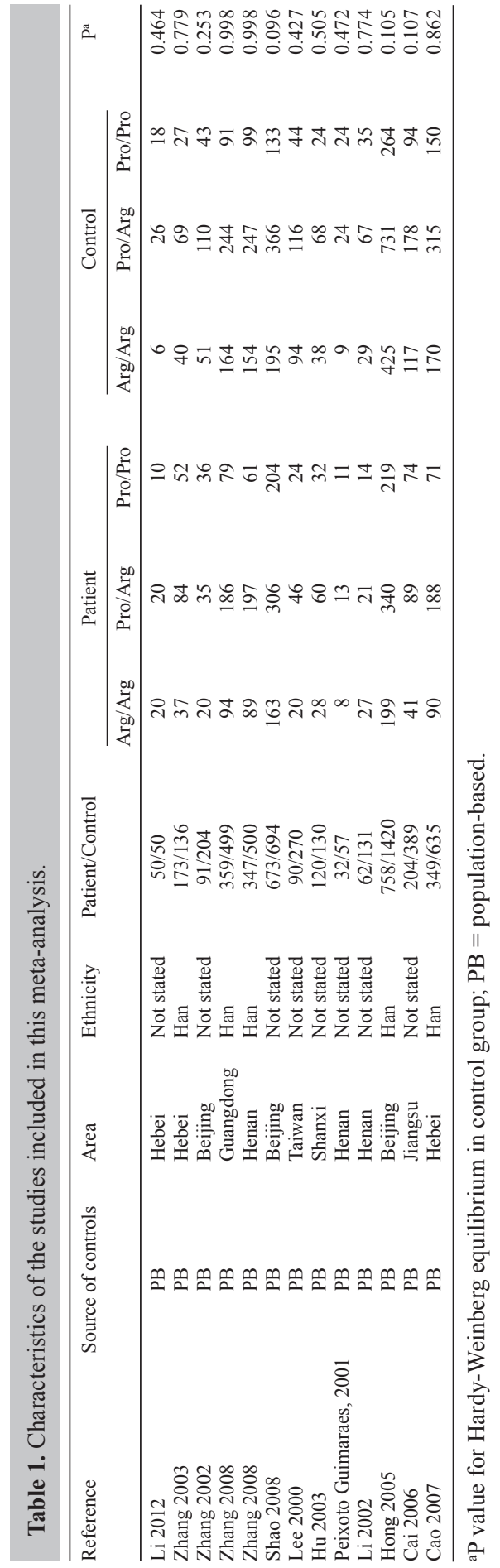




\section{Quantitative data synthesis}

The main results of this meta-analysis and of the heterogeneity test are shown in Table 2. In the overall analyses, the Pro/Pro genotype was shown to be significantly related to EC risk under the allele, recessive, homozygous, and heterozygous models (Pro vs Arg: OR = 1.17, $95 \% \mathrm{CI}=1.01-1.34$; Pro/Pro vs Arg/Arg+Arg/Pro: $\mathrm{OR}=1.32,95 \% \mathrm{CI}=1.06-1.66$; Pro/ Pro vs Arg/Arg: OR = 1.35, 95\%CI = 1.03-1.78; Pro/Pro vs Arg/Pro: $\mathrm{OR}=1.32,95 \% \mathrm{CI}=$ 1.06-1.65). However, no statistically significant associations were found under the dominant model (Pro/Pro+Arg/Pro vs Arg/Arg: OR $=1.16,95 \% \mathrm{CI}=0.95-1.41$ ).

In the subgroup analysis based on geographical location, the results showed that carrying the Pro/Pro genotype and the Pro allele was significantly related to EC risk among the populations from Southern China (Pro vs Arg: OR = 1.39, 95\%CI = 1.21-1.60; Pro/Pro+Arg/ Pro vs Arg/Arg: $\mathrm{OR}=1.57,95 \% \mathrm{CI}=1.26-1.96$; Pro/Pro vs Arg/Arg+Arg/Pro: OR $=1.52$, 95\%CI = 1.21-1.90; Pro/Pro vs Arg/Arg: OR = 1.89, 95\%CI = 1.43-2.49; Pro/Pro vs Arg/Pro: $\mathrm{OR}=1.32,95 \% \mathrm{CI}=1.04-1.69$ ), but not among those from Northern China (Table 2). Upon further subgroup analysis by ethnicity, the pooled results showed that there was significant association between p53 codon 72 polymorphism and EC risk among Han Chinese (Pro vs Arg: $\mathrm{OR}=1.18,95 \% \mathrm{CI}=1.02-1.36$; Pro/Pro + Arg $/$ Pro $v s \mathrm{Arg} / \mathrm{Arg}$ : OR = 1.23, 95\%CI = 1.09-1.40; Pro/Pro vs Arg/Arg: OR $=1.37,95 \% \mathrm{CI}=1.01-1.86)$ and among the Chinese with no stated ethnicity (Pro/Pro vs Arg/Pro+Arg/Arg: OR = 1.43, 95\%CI = 1.05-1.93; Pro/Pro vs Arg/Pro: $\mathrm{OR}=1.64,95 \% \mathrm{CI}=1.38-1.95)($ Table 2$)$.

\section{Sensitivity analysis}

In order to compare the differences within and evaluate the sensitivity of the metaanalyses, we used both the fixed- and random-effect models to evaluate the stability of the meta-analysis. None of the results was materially altered in the overall and subgroup analysis (data not shown). Hence, results of the sensitivity analysis suggested that the data in this metaanalysis were relatively stable and credible.

\section{Bias diagnosis}

The Begg's funnel plot and the Egger test were performed to assess the publication bias of the literature. The shape of the funnel plots did not reveal any evidence of obvious asymmetry. The Egger test also showed that there was no significantly statistical evidence of publication bias for any of the genetic models in the overall analysis (allele model: $\mathrm{t}=-1.38$, $\mathrm{P}=0.194$; dominant model: $\mathrm{t}=-1.33, \mathrm{P}=0.210$; recessive model: $\mathrm{t}=-1.34, \mathrm{P}=0.207$; homozygous model: $\mathrm{t}=-1.54, \mathrm{P}=0.153$; heterozygous model: $\mathrm{t}=-0.88, \mathrm{P}=0.399$ ). 


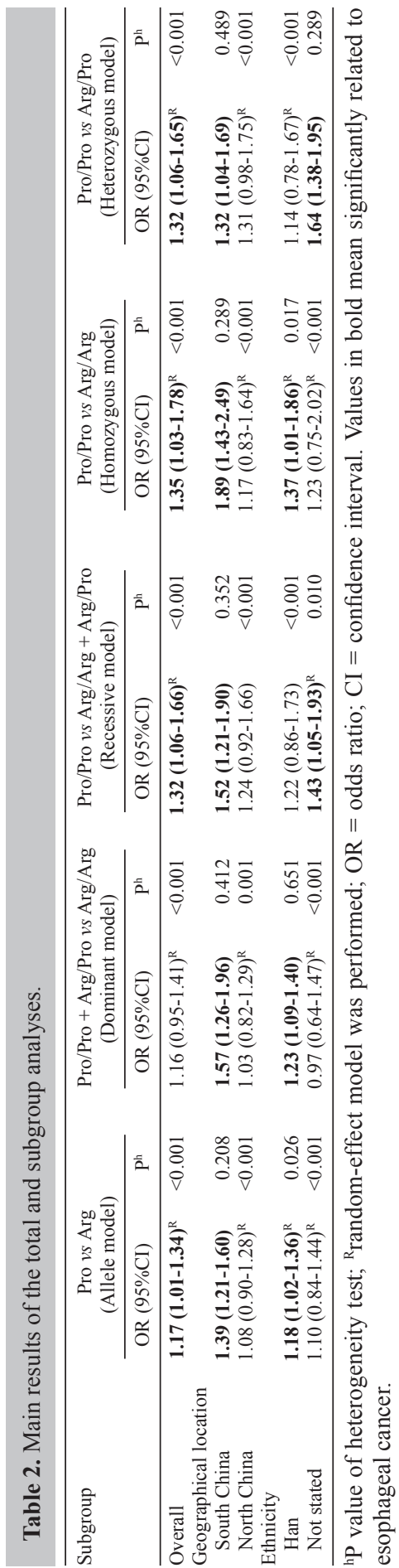




\section{DISCUSSION}

There is a wide variation in the incidence of EC among different regions, and this sharp variation across the different areas suggests that environmental factors together with genetic factors play important roles in the development of EC (Zheng et al., 2011). However, only a minor proportion of the populations exposed to the known risk factors including smoking tobacco and drinking alcohol develops EC, which suggests that genetic factors might play a very important role in the development of EC (Zhong et al., 2013). Currently, numerous genetic polymorphisms have been suggested to be associated with risk of EC, such as the GSTM1 polymorphism (Zhong et al., 2013). The findings from previous studies on the association between genetic polymorphisms and EC risk further demonstrate the important role of genetic susceptibility in the development of EC.

Several previous studies have investigated p53 codon 72 polymorphism and its association with EC in the Chinese population; however, definitive conclusions could not be drawn. Most of those studies involved few patients, and the small sample size limited the reliability of determining the genetic effect. To provide a more precise estimation on the association between p 53 codon 72 polymorphism and the risk of EC in the Chinese population, we performed a meta-analysis involving 12 articles related to 553 codon 72 polymorphism and EC. Meta-analysis results showed that the p53 codon 72 polymorphism was significantly associated with EC risk among Chinese populations. The sensitivity analysis confirmed the reliability and stability of the meta-analysis, and no publication bias was found among the studies using the Egger test. Therefore, the findings from our meta-analysis provide strong evidence for the association between p53 codon 72 polymorphism and the risk of EC in Chinese populations, and in particular for the contribution of the Pro/ Pro genotype to the increased risk of EC in Chinese. Our results were consistent with previously published meta-analyses (Wang et al., 2010; Jiang et al., 2011; Zhao et al., 2010, 2013). However, these meta-analyses did not search Chinese databases (CBM and CNKI), and included a smaller number of studies conducted in Chinese populations than did our meta-analysis.

Furthermore, when we performed the subgroup analyses by geographical location and ethnicity, significant association with the susceptibility for developing EC was found in Southern China, in Han Chinese and in the population without stated ethnicity. This is the first review that reports geographical location as an important marker in the population genetic background for the influence of the p53 codon 72 polymorphism in China. Despite the limited sample size (only three studies on Southern China), our data indicated that the Pro/Pro genotype represents a susceptibility risk marker for the development of EC in Southern but not in Northern China. However, whether the p53 codon 72 genotype represents a good biomarker for therapeutic effects in patients with EC has not been well evaluated and needs further studies. In addition, the association between p53 codon 72 genotype and risk of EC in other populations is still unclear, and more case-control studies with large sample sizes are needed.

In conclusion, although studies investigating the association between p53 codon 72 polymorphism and the risk of EC in China have previously arrived at different conclusions, this meta-analysis suggests that $\mathrm{p} 53$ codon 72 polymorphism might be associated with EC in Chinese populations, especially in Southern China.

\section{ACKNOWLEDGMENTS}

Research supported by the Science Foundation of Guangdong Province (to J.Z. Peng, \#2011B0613000167). 


\title{
Conflicts of interest
}

\author{
The authors declare no conflict of interest.
}

\section{REFERENCES}

Cai L, Mu LN, Lu H, Lu QY, et al. (2006). Dietary selenium intake and genetic polymorphisms of the GSTP1 and p53 genes on the risk of esophageal squamous cell carcinoma. Cancer Epidemiol. Biomarkers Prev. 15: 294-300.

Cao YY, Ge H, Chen LQ, Chen ZF, et al (2007). Correlation of 53BP1 and p53 polymorphisms to susceptibility to esophageal squamous cell carcinoma and gastric cardiac adenocarcinoma. Ai Zheng 26: 1052-1057.

Dumont P, Leu JI, Della Pietra AC 3rd, George DL, et al. (2003). The codon 72 polymorphic variants of p53 have markedly different apoptotic potential. Nat. Genet. 33: 357-365.

Higgins JP and Thompson SG (2002). Quantifying heterogeneity in a meta-analysis. Stat. Med. 21: 1539-1558.

Hong Y, Miao X, Zhang X, Ding F, et al. (2005). The role of P53 and MDM2 polymorphisms in the risk of esophageal squamous cell carcinoma. Cancer Res. 65: 9582-9587.

$\mathrm{Hu}$ N, Li WJ, Su H, Wang C, et al. (2003). Common genetic variants of TP53 and BRCA2 in esophageal cancer patients and healthy individuals from low and high risk areas of northern China. Cancer Detect. Prev. 27: 132-138.

Huang GL, Wang SK, Su M, Wang TT, et al. (2013). Serum folate, MTHFR C677T polymorphism and esophageal squamous cell carcinoma risk. Biomed. Environ. Sci. 26: 1008-1012.

Jemal A, Bray F, Center MM, Ferlay J, et al. (2011). Global cancer statistics. CA Cancer J. Clin. 61: 69-90.

Jiang DK, Yao L, Wang WZ, Peng B, et al. (2011). TP53 Arg72Pro polymorphism is associated with esophageal cancer risk: a meta-analysis. World J. Gastroenterol. 17: 1227-1233.

Langerød A, Bukholm IR, Bregârd A, Lønning PE, et al. (2002). The TP53 codon 72 polymorphism may affect the function of TP53 mutations in breast carcinomas but not in colorectal carcinomas. Cancer Epidemiol. Biomarkers Prev. 11: 1684-1688.

Lee JM, Lee YC, Yang SY, Shi WL, et al. (2000). Genetic polymorphisms of $p 53$ and GSTP1, but not NAT2, are associated with susceptibility to squamous-cell carcinoma of the esophagus. Int. J. Cancer 89: 458-464.

Levine AJ (1997). p53, the cellular gatekeeper for growth and division. Cell 88: 323-331.

Li Q (2012). Research on mutations/polymorphism of P53 4th exon codon $72 \mathrm{Arg} / \mathrm{Pro}$ and the associated pathogenic factors for esophageal cancer patients in Hebei (article in Chinese). Master's thesis, Hebei Medical University, Hebei.

Li T, Lu ZM, Guo M, Wu QJ, et al. (2002). p53 codon 72 polymorphism (C/G) and the risk of human papillomavirusassociated carcinomas in China. Cancer 95: 2571-2576.

Lin Y, Totsuka Y, He Y, Kikuchi S, et al. (2013). Epidemiology of esophageal cancer in Japan and China. J. Epidemiol. 23: $233-242$.

Peixoto Guimaraes D, Hsin Lu S, Snijders P, Wilmotte R, et al. (2001). Absence of association between HPV DNA, TP53 codon 72 polymorphism, and risk of oesophageal cancer in a high-risk area of China. Cancer Lett. 162: 231-235.

Shao Y, Tan W and Zhang S (2008). P53 gene codon 72 polymorphism and risk of esophageal squamous cell carcinoma: a case/control study in a Chinese population. Dis. Esophagus 21: 139-143.

Thomas M, Kalita A, Labrecque S, Pim D, et al. (1999). Two polymorphic variants of wild-type p53 differ biochemically and biologically. Mol. Cell Biol. 19: 1092-1100.

Wang B, Wang D, Zhang D, Li A, et al. (2010). Pro variant of TP53 Arg72Pro contributes to esophageal squamous cell carcinoma risk: evidence from a meta-analysis. Eur. J. Cancer Prev. 19: 299-307.

Wiseman M (2008). The second World Cancer Research Fund/American Institute for Cancer Research expert report. Food, nutrition, physical activity, and the prevention of cancer: a global perspective. Proc. Nutr. Soc. 67: 253-256.

Zhang J (2008). Association of p53 Pro72Arg polymorphism with susceptibility to esophageal \& cardiac cancer in south and north of China (article in Chinese). Master's thesis, Shantou University, Shantou.

Zhang JH, Li Y, Wang R, Wen DG, et al. (2003). p53 gene polymorphism with susceptibility to esophageal cancer and lung cancer in Chinese population (article in Chinese). Zhonghua Zhong Liu Za Zhi 25: 365-367.

Zhang L, Xing D, He Z and Lin D (2002). p53 gene codon 72 polymorphism and susceptibility to esophageal squamous cell carcinoma in a Chinese population (article in Chinese). Zhonghua Yi Xue Yi Chuan Xue Za Zhi 19: 10-13.

Zhao L, Zhao X, Wu X and Tang W (2013). Association of p53 Arg72Pro polymorphism with esophageal cancer: a metaanalysis based on 14 case-control studies. Genet. Test. Mol. Biomarkers 17: 721-726.

Zhao Y, Wang F, Shan S, Zhao Y, et al. (2010). Genetic polymorphism of $p 53$, but not GSTP1, is association with susceptibility to esophageal cancer risk - a meta-analysis. Int. J. Med. Sci. 7: 300-308. 
Zheng S, Vuitton L, Sheyhidin I, Vuitton DA, et al. (2011). Northwestern China: a place to learn more on oesophageal cancer. Part two: gene alterations and polymorphisms. Eur. J. Gastroenterol. Hepatol. 23: 1087-1099.

Zhong S, Zhao W, Lu C, Li B, et al. (2013). Glutathione S-transferase M1 null genotype contributes to increased risk of esophageal carcinoma in Chinese population. Tumour Biol. 34: 2403-2407. 